\title{
A Comparison of Two Recording Montages for Ocular Vestibular Evoked Myogenic Potentials in Patients with Superior Canal Dehiscence Syndrome
}

DOI: $10.3766 /$ jaaa.17093

\author{
Kathryn Makowiec* \\ Devin McCaslin $\dagger$ \\ Kelsey Hatton*
}

\begin{abstract}
Objective: The purpose of this investigation was to evaluate the sensitivity and specificity of the ocular vestibular evoked myogenic potential (oVEMP) using two electrode montages in patients with confirmed unilateral superior semicircular canal dehiscence syndrome (SCDS).

Study Design: This study evaluated oVEMP response characteristics measured using two different electrode montages from 12 unilateral SCDS ears and 36 age-matched control ears (age range = 23-66). The oVEMP responses were elicited using $500 \mathrm{~Hz}$ tone-burst air conduction stimuli presented at an intensity of $95 \mathrm{~dB} \mathrm{nHL}$ and a rate of $5.1 / \mathrm{sec}$. The two electrode montages used are described as an "infraorbital" montage and a "belly-tendon" montage.

Setting: Balance function laboratory embedded in a large, tertiary care otology clinic.

Results: The belly-tendon electrode montage resulted in significantly larger amplitude responses than the infraorbital electrode montage for the ears with SCDS and the normal control ears. For both electrode montages the ear with SCDS exhibited a significantly larger amplitude response, $\sim 50 \%$ larger than the response amplitude from the normal control ear. The belly-tendon montage additionally produced larger median increases in amplitude compared with the infraorbital montage. Specifically, the median increase in oVEMP N1-P1 amplitudes using the belly-tendon montage was 39\% greater in control ears, $76 \%$ greater in the SCDS ears, and $17 \%$ greater in the contralateral SCDS ears.
\end{abstract}

Conclusions: The belly-tendon electrode montage yields significantly larger oVEMP amplitude responses for participants with SCDS and normal control participants.

Key Words: vestibular, utricle, oVEMP, superior canal dehiscence, SCDS

Abbreviations: $\mathrm{CT}=$ computed tomography; cVEMP = cervical vestibular evoked myogenic potential; oVEMP = ocular vestibular evoked myogenic potential; SCDS = superior semicircular canal dehiscence syndrome; SD = standard deviation; VEMP = vestibular evoked myogenic potential

\section{BACKGROUND}

$\mathrm{T}$ he membranous labyrinth of the inner ear is filled with fluid and encased in the bone of the otic capsule. A normal-functioning inner ear has two openings in this bone, the oval and round windows, to facilitate the transfer of sound energy to the inner ear. However, patients with superior semicircular canal de- hiscence syndrome (SCDS) have a pathological third opening in the bone of the otic capsule in an area that typically covers the superior semicircular canal (Minor et al, 1998). Patients identified with this additional opening or "third window" will report auditory, vestibular, and/or visual symptoms in response to sound.

The initial description of SCDS was by Minor et al (1998). The disorder is characterized by a variety of

*Division of Vestibular Sciences, Department of Hearing and Speech Sciences, Vanderbilt University Medical Center, Nashville, TN; $†$ Dizziness and Balance Disorders Clinic, Mayo Clinic, Rochester, MN

Corresponding author: Kathryn Makowiec, Division of Vestibular Sciences, Department of Hearing and Speech Sciences, Vanderbilt University Medical Center, Medical Center East, Nashville, TN 37232-8025; Email: kathrynmakowiec@gmail.com

Funding for this research project was provided by the American Academy of Audiology Foundation as part of a Student Researcher Grant. 
vestibular and visual symptoms, such as vertigo, that can be evoked by either sound, changes in middle ear pressure, and/or changes in intracranial pressure. Patients with SCDS often report oscillopsia or dizziness when coughing, sneezing, or straining. In addition, they may present with auditory symptoms such as autophony, conductive hearing loss with normal immittance, and hypersensitivity to bone-conducted sounds (Minor et al, 1998). Although the gold standard confirmatory test is a high-resolution computed tomography (CT) scan with fine cuts, audiological tests can be used to corroborate the presence of SCDS and to evaluate if the SCDS is active and symptomatic. To date, pure-tone audiometry and vestibular evoked myogenic potential testing (VEMPs) have been shown to be clinically useful in this population (Brantberg et al, 1999; Belden et al, 2003; Hunter et al, 2016). Both the sensitivity and specificity of the ocular VEMP (oVEMP) and cervical VEMP (cVEMP) have been investigated for identifying SCDS (Zuniga et al, 2013). The cVEMP is a short latency ( $\sim 13$ msec) evoked myogenic potential recorded from the sternocleidomastoid muscle (Colebatch et al, 1994). The oVEMP is a short latency ( $\sim 10 \mathrm{msec})$ evoked myogenic potential recorded from the extraocular muscles (Rosengren et al, 2005; Todd et al, 2007). Both VEMP tests are well tolerated by patients and simple to administer. The majority of evidence now suggests that the cVEMP measured from the ipsilateral sternocleidomastoid muscle using mechanical or air-conducted stimuli is generated by the saccule (Colebatch et al, 1994). The receptor organ of the oVEMP measured in response to mechanical and air-conducted stimuli is now believed to be the utricle (Govender et al, 2009; Iwasaki et al, 2009; Manzari et al, 2010; Welgampola and Carey, 2010; Curthoys et al, 2011). The oVEMP response is recorded with electrodes beneath both eyes, but the largest response is typically seen in the contralateral eye muscle recording (Chihara et al, 2007; Iwasaki et al, 2007; Curthoys et al, 2011; Murnane et al, 2011). The response originates from the utricle and superior portion of the vestibular nerve, travels along the medial longitudinal fasciculus, and terminates on the ipsilateral superior rectus muscle and contralateral inferior oblique muscle (Rosengren et al, 2010). The evoked potential of the contralateral inferior oblique muscle is best recorded by an electrode placed beneath the lower eyelid while having the participant look supramedial (Suzuki et al, 1969; Dumitru and DeLisa, 1991; Chihara et al, 2007; Todd et al, 2007; Govender et al, 2009; Rosengren et al, 2009).

Various stimulating and recording parameters have been evaluated to determine how to maximize the sensitivity and specificity of the VEMP response for identifying SCDS. For example, a study by Zuniga et al (2013) examined the effectiveness of tone burst and click evoked oVEMPs and cVEMPs for diagnosing SCDS. They examined the responses of 29 ears with SCDS and 25 age-matched control participants and calculated decade-specific peak-to-peak amplitude values to use clinically as a cutoff for requiring further examination. The authors provided decade-specific cutoff values for their cohort and found excellent $(>90 \%)$ sensitivity and specificity to SCDS using $500 \mathrm{~Hz}$ tone-burst stimuli for ages 30-70.

In addition to investigations describing the best recording and stimulating parameters for identifying SCDS using the oVEMP, there have also been several investigations of analysis methods. Absolute oVEMP N1-P1 peak-to-peak amplitude has been shown to be a useful measure in identifying patients with SCDS. Piker et al (2011) found the peak-to-peak amplitude across all ages to be $4.4 \mu \mathrm{V}$ and defined the upper limit of oVEMP interaural amplitude asymmetry as $34 \%$. Zuniga et al (2014) compared oVEMP amplitudes in patients with SCDS and an age-matched control group using two electrode montages. The first electrode montage used an active (noninverting) electrode placed $\sim 3 \mathrm{~mm}$ below each eye, a reference (inverting) electrode placed $2 \mathrm{~cm}$ below each active electrode, and the ground on the sternum. The second montage used an active electrode placed $\sim 3 \mathrm{~mm}$ below each eye, a single reference electrode placed on the chin, and the ground on the sternum. The median peak-to-peak amplitude for SCDS ears in this study was $66.6 \mu \mathrm{V}$ with the infraorbital electrode montage and $72.2 \mu \mathrm{V}$ when using an electrode montage with the reference located on the chin. The median peak-to-peak amplitude for the normal ear contralateral to the SCDS ear was $4.55 \mu \mathrm{V}$ with the infraorbital electrode montage and $7.7 \mu \mathrm{V}$ when using the chin for reference. A clinical cutoff value for differentiating normal participants to those with SCDS was identified as $24.5 \mu \mathrm{V}$ or greater for absolute N1-P1 peak-to-peak amplitude when using the infraorbital electrode montage and a cutoff value of $30.8 \mu \mathrm{V}$ or greater when using the chin for reference (Zuniga et al, 2014).

The variability caused by using different electrode montages has been studied. The vast majority of clinics favor an electrode montage reported in early articles describing the oVEMP (Chihara et al, 2007). The authors reported that the noninverting (active) electrode was placed infraorbitally and centered at the margin of the lower eyelid. The inverting (reference) electrode was placed $2 \mathrm{~cm}$ inferior to the location of the noninverting electrode (Chihara et al, 2007; Todd et al, 2007; Govender et al, 2009). This is referred to as the "infraorbital" montage. A second electrode montage was reported by Sandhu et al (2013). They recommend the noninverting (active) electrode was placed at the margin of the lower eyelid slightly lateral to midline, and the inverting (reference) electrode was placed rostral to the inner canthus of the eye. This electrode montage is referred to as the "bellytendon" montage. Sandhu et al (2013), Vanspauwen et al (2017), and Makowiec et al (2017) obtained significantly larger amplitude responses measured with the belly-tendon montage in otologically normal participants 
compared with amplitudes measured with the infraorbital montage. This belly-tendon electrode montage is felt to be optimal because of the lessened likelihood of reference contamination as the inverting electrode is placed over a tendon, which is believed to be electrically neutral (Piker et al, 2011).

Although historically oVEMPs have been measured with the participant in the supine position, recent studies by Taylor et al (2015) and Makowiec et al (2017) found higher amplitude responses when the participant was in the sitting position. The theoretical explanation as to why the sitting position would result in larger amplitude responses is that there is less baseline stimulation of the utricle when the participant is sitting, so the application of a sudden, high-intensity stimulus would result in greater excitation. When the participant is in the supine position, the mass of the otoliths may reduce the maximum response that can be obtained (Shojaku et al, 2008; Wang et al, 2014; Taylor et al, 2015). Data was collected for this study with the participants in the sitting position, as the response characteristics of the oVEMP in SCDS has yet to be investigated in the sitting position.

With confirmed reports of enhanced N1-P1 amplitudes using the belly-tendon montage in normal participants, there is interest in revisiting the assessment of peak-to-peak oVEMP amplitudes for patients with SCDS. As previous studies examining the peak-to-peak amplitude and sensitivity and specificity of the oVEMP response in SCDS have not included the belly-tendon montage (reported by Sandhu et al), the effect this montage would have on the oVEMP amplitudes of patients with SCDS is unknown. The purpose of the present investigation was to examine the effects of different electrode montages in patients with unilateral SCDS. We compared these responses with each participant's unaffected ear in addition to the ears of age-matched, otologically normal control participants. We hypothesized that the belly-tendon montage would result in larger peak-topeak amplitudes for the ears with SCDS than the normal control ears and would result in larger peak-to-peak amplitudes than the traditional infraorbital electrode montage for both ears with SCDS and normal control ears.

\section{METHODS}

\section{Participants}

All procedures were approved by the Vanderbilt University Medical Center's Human Research Protection Program and Institutional Review Board (IRB\# 140805). Participants included two groups of participants, split into three categories listed in the following paragraphs. The groups were composed of 12 unilateral ears diagnosed with SCDS [age range $=25-68$ years, mean $=48.91$, standard deviation (SD) = 15.56], 12 ears contralateral to the SCDS ear, and 24 age-matched ( \pm 3 years) control participants with normal hearing and no history of dizziness and/ or imbalance or any other otologic abnormalities (age range $=22-68$, mean $=47.16, \mathrm{SD}=13.76$ ). The SCDS was confirmed for each participant via temporal bone CT scan before participation in the study. Participants with SCDS were identified through medical record review; 8 of the 12 participants had previously undergone balance function testing showing abnormal VEMPs and the SCDS was later confirmed through CT scan. The remaining four participants were identified after CT scan and Ear Nose and Throat evaluation at which time they reported symptoms consistent with SCDS.

Each SCDS ear was compared with the unaffected ear within the same participant and to the ears of an agematched, normal hearing control. All participants underwent hearing evaluations with pure-tone air and bone conduction audiometry, and the age-matched controls all had normal hearing sensitivity from 250 to $8000 \mathrm{~Hz}$ without any air-bone gaps. Subjective symptoms were recorded for each participant with SCDS before data collection. Nine of the 12 participants reported dizziness with straining, 11 of the 12 participants reported abnormal auditory perceptions, and 11 of the 12 participants reported autophony. Dizziness Handicap Inventory (DHI) scores for the participants with SCDS ranged from 0 to 68 . DHI scores for the normal control participants were all 0 .

\section{Procedures}

All participants were seated in a comfortable chair. Electrodes were applied using a conventional clean electrode preparation technique. Disposable silver/silver chloride electrodes were used for testing. Absolute electrode impedances were $<5,000 \Omega$ with interelectrode impedances of $<2,000 \Omega$. The active electrodes were placed $1 \mathrm{~cm}$ inferior to each eye centered at the orbital midline for the infraorbital electrode montage, and $1 \mathrm{~cm}$ inferior to each eye but lateral to the orbital midline for the bellytendon montage. The reference electrodes were placed $2-3 \mathrm{~cm}$ inferior to the active electrode for the infraorbital electrode montage and at the inner canthus of each eye for the belly-tendon electrode montage. The ground electrode was placed at Fpz (forehead) for both montages (Figure 1, originally published in Makowiec et al, 2017). Each participant was asked to focus on a fixed visual target where their gaze was elevated $30^{\circ}$ above a neutral position of midline gaze.

When recording the oVEMP, the participants were seated and instructed to keep their head stable at midline and maintain their gaze at a fixed target positioned $30^{\circ}$ upward at midline. Responses were recorded with the participants in the sitting position (Makowiec et al, 2017). Stimuli for the oVEMP recordings were presented monaurally through an Etymotic ER-3A insert earphone and consisted of $500 \mathrm{~Hz}$ tone bursts presented at $95 \mathrm{~dB}$ $\mathrm{nHL}$ with a rate of $5.1 / \mathrm{sec}$ and a two-cycle risetime, one-cycle plateau, and a two-cycle falltime. An Intelligent 


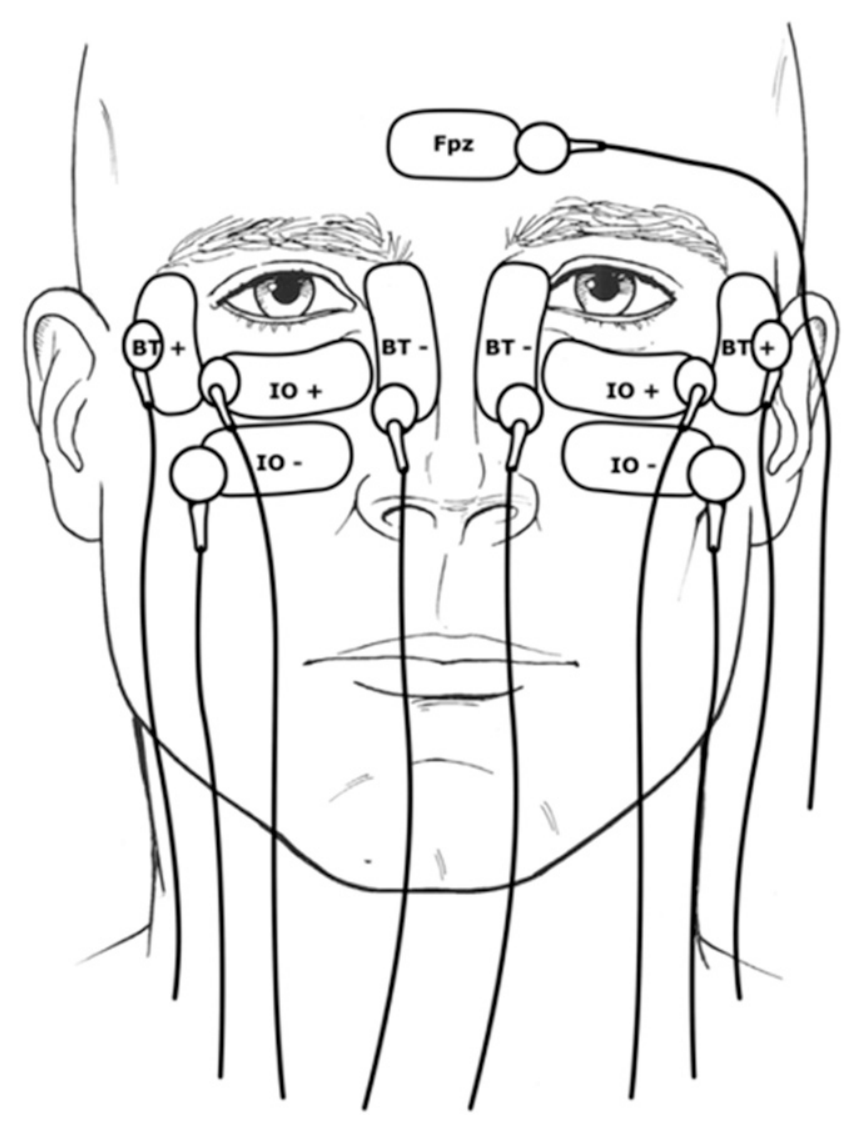

Figure 1. Electrode montages for testing; BT + represents bellytendon active, BT - represents belly-tendon reference, IO+ represents infraorbital active, and IO- represents infraorbital reference.

Hearing Systems-evoked potential recording system was used to simultaneously record the oVEMP response from beneath the contralateral eye from each of the two electrode montages. Electromyography activity was amplified by 100,000 times and the signal averaged over $100 \mathrm{msec}$. A minimum of 160 individual samples were collected for each recording. Each tracing was replicated at least one time so that the waveform reproducibility could be confirmed. The participants were given 2-3 min to rest their eyes between recordings. Order effects were eliminated by counterbalancing the order of the starting ear. oVEMPs from the two electrode montages were recorded simultaneously, so counterbalancing the electrode montage was unnecessary.

\section{Statistical Analysis}

Statistical procedures were performed on SPSS 23 for Mac (Apple, Cupertino, CA). The means and SDs for the N1-P1 peak-to-peak oVEMP amplitudes, N1 latency, and P1 latency responses were calculated for both electrode montages. Only the response from the contralateral eye was recorded and analyzed (Rosengren et al, 2005; Todd et al, 2007; Makowiec et al, 2017). Kruskal-Wallis one-way repeated measures analysis of variance was used to detect differences in oVEMP response characteristics between the three groups (SCDS ear, contralateral ear to the SCDS, and normal control ear). P1 latency, N1 latency, and N1-P1 peak-to-peak amplitudes were assigned as dependent variables and the recording technique was assigned as the independent variable. The difference in oVEMP amplitude obtained with each montage was calculated as a change expressed in percentage as described by Zuniga et al (2014): [(infraorbital - belly-tendon)/belly-tendon]. Differences in N1-P1 peak-peak amplitude between montages were analyzed for all groups using the Wilcoxon signed-rank test. Results were considered significant at the $p<0.05$ level.

\section{RESULTS}

\section{oVEMP N1-P1 Peak-to-Peak Amplitude Findings}

oVEMP N1-P1 recordings with the belly-tendon montage generated significantly different amplitudes across the three ear groups $[H(2)=34.29, p<0.001]$. Post hoc analysis showed that oVEMP N1-P1 amplitudes were significantly larger in ears with SCDS (mean $=20.10$ $\mu \mathrm{V}$ ) compared with controls (mean $=4.1 \mu \mathrm{V} ; U=$ $126, p<0.001$ ) and with the normal ears contralateral to the SCDS ears (mean $=2.7 \mu \mathrm{V} ; U=22, p<0.001$; Figure 2). There was no significant difference in N1-P1 amplitudes between the normal ears contralateral to the SCDS ears and the control ears $(U=251, p=$ 0.155). Sample oVEMP waveforms obtained using both electrode montages from the three ear categories are shown in the figure below (Figure 3).

oVEMP N1-P1 recordings with the infraorbital montage generated significantly different $\mathrm{N} 1$ amplitudes across the three ear groups $[H(2)=17.20, p<0.001]$. Post hoc analysis showed that oVEMP N1-P1 amplitudes were significantly larger in ears with SCDS (mean $=7.98 \mu \mathrm{V}$ ) compared with controls (mean $=2.49 \mu \mathrm{V} ; U=175$, $p<0.001)$ and the normal ears contralateral to the SCDS ears (mean $=2.52 \mu \mathrm{V} ; U=40, p<0.001$; Figure 2 ). There was no significant difference in N1-P1 amplitudes between the normal ears contralateral to the SCDS ears and the control ears $(U=245, p=0.625)$.

\section{oVEMP N1 and P1 Latency Findings}

oVEMP N1 and P1 latencies recorded with both electrode montages were analyzed for all three subgroups. The average $\mathrm{N} 1$ and $\mathrm{P} 1$ latencies for both electrode montages and all three subgroups are shown in Table 1. Post hoc analysis showed that there were no significant differences in N1 or P1 latency between any of the ear groups (Figures 4 and 5).

\section{The Effect of Montage on Amplitude}

Collapsed peak-to-peak N1-P1 amplitudes with bellytendon montage were larger than those using the 
N1-P1 Amplitude-montage

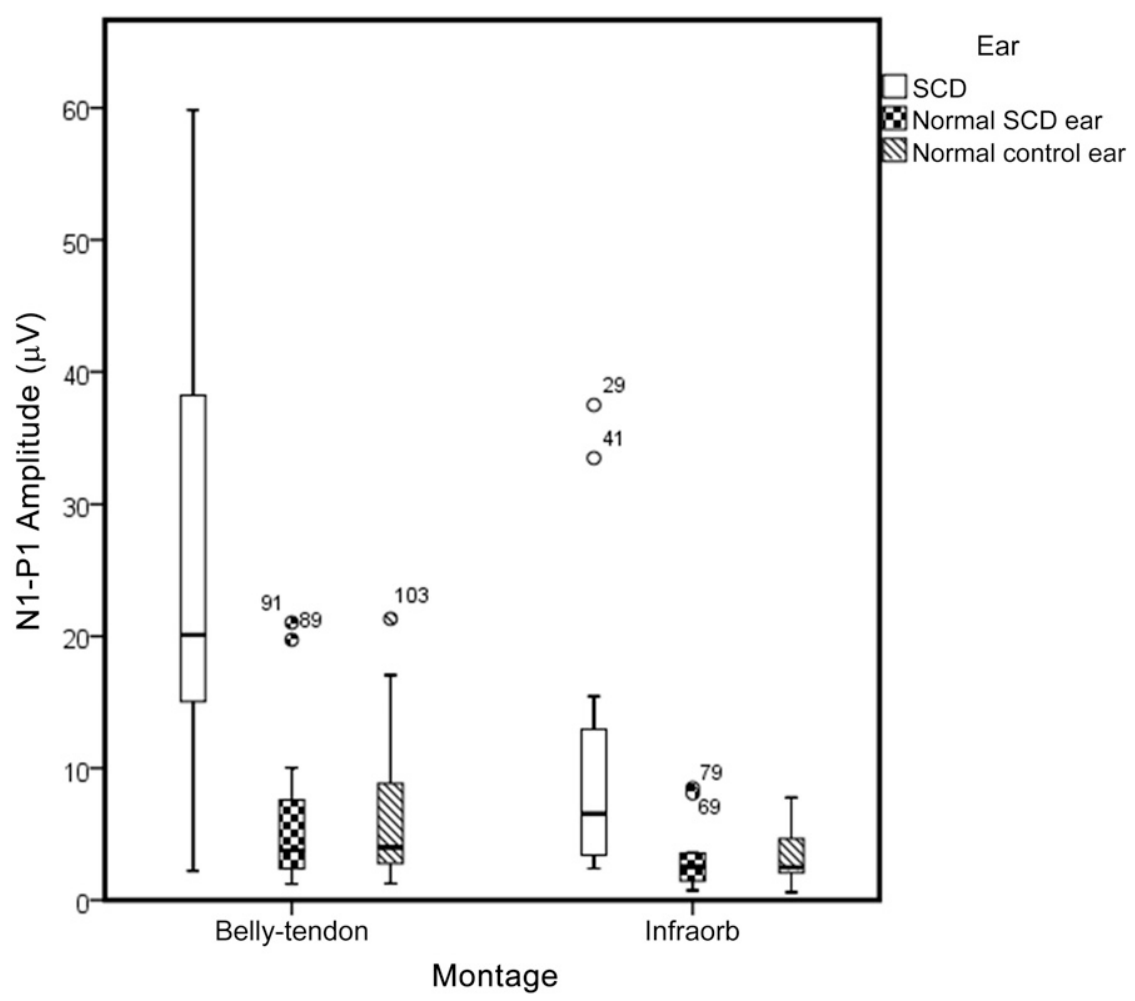

Figure 2. Boxplots of N1-P1 amplitude for the two different montages and three groups. The thin line within the box designates the mean. The error bars above and below the box indicate the 90th and 10th percentiles. Outliers are represented by the points outside the boxes.

infraorbital when all groups were combined. The N1-P1 peak-to-peak amplitudes in the SCDS ears were significantly larger when recording with the belly-tendon electrode montage than when recording with the infraorbital electrode montage $(Z=-3.89, p<0.001)$. The N1-P1 peak-to-peak amplitudes in the normal ear contralateral to the SCDS ears recorded using the belly-tendon montage were significantly larger than the responses obtained using the infraorbital montage $(Z=-2.90, p=0.004)$. The N1-P1 peak-to-peak amplitudes in the normal control ears recorded using the belly-tendon montage were significantly larger than the responses obtained using the infraorbital montage $(Z=-4.13, p<0.001)$. The bellytendon montage produced larger median increases in amplitude compared with the infraorbital montage. Specifically, the median increase in oVEMP N1-P1 amplitudes using the belly-tendon montage was $39 \%$ greater in control ears (range $=100.51 \%$ ), $76 \%$ greater in the SCDS ears (range $=74 \%$ ), and $17 \%$ greater in the contralateral SCDS ears $($ range $=100 \%)$. The average N1-P1 peak-to-peak amplitudes for both electrode montages and all three subgroups are shown in Table 2.

\section{DISCUSSION}

linically, the oVEMP is a useful tool in screening for patients with SCDS, and a large amplitude
oVEMP response can serve as a "red flag" for this disorder. Patients with unilateral SCDS typically generate significantly larger amplitude responses in the affected ear when compared with their normal ear. This study examined the effect of two electrode montages on oVEMP N1-P1 peakto-peak amplitude, N1 latency, and P1 latency in a cohort of patients with radiologically confirmed SCDS. Responses from ears with SCDS were compared with responses from the non-SCDS ears of each participant and the ears of agematched, normal hearing control participants.

Piker et al (2011) found reference contamination when using the traditional infraorbital electrode montage to record oVEMP responses in normal participants. This resulted in artificially reduced oVEMP peak-to-peak amplitude responses. They found that when the inverting/reference electrode is placed outside of the electrical field of the evoked potential (i.e., on an electrically neutral location), the oVEMP amplitudes were significantly enhanced. Zuniga et al (2014) studied the effect of electrode placement for a cohort of participants with SCDS and found results that were in agreement with the Piker et al (2011) study. Their results showed oVEMP amplitudes increased by moving the inverting/reference electrode further away from the active/noninverting electrode, thus reducing the likelihood of reference contamination occurring.

Piker et al and Zuniga et al both compared the traditional infraorbital electrode montage to an electrode 

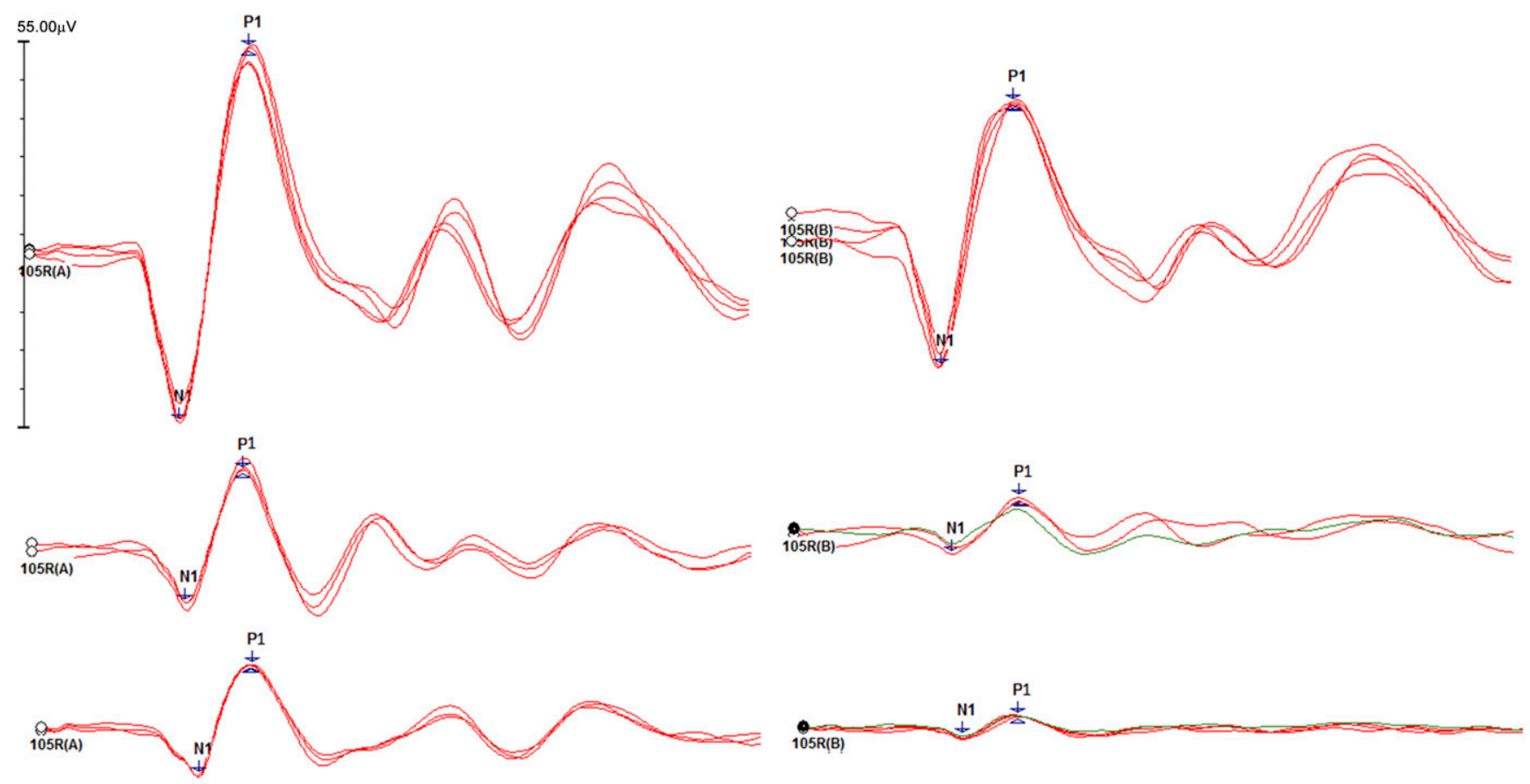

Figure 3. Sample oVEMP waveforms obtained from both electrode montages for all three category groups. Left-hand side $=$ responses from the belly-tendon electrode montage, and right-hand side = responses from the infraorbital electrode montage. Top to bottom: SCDS ear, normal ear contralateral to the SCDS ear, and normal control ear. (This figure appears in color in the online version of this article.)

montage with the active/noninverting electrodes beneath the eyes and the reference/inverting electrode on the chin. The study by Sandhu et al (2013) introduced a bellytendon electrode montage which was shown to result in significantly larger amplitude oVEMP responses in otologically normal participants. This finding of increased amplitude responses when using the belly-tendon electrode montage was confirmed by Vanspauwen et al (2017) and Makowiec et al (2017). Such studies confirm the belief that reference contamination can result in significantly reduced amplitude responses and contamination is attributed to the location of the reference electrode relative to the active electrode. Before the current study the belly-tendon electrode montage had not been evaluated in patients with SCDS.

As predicted, this study found the affected SCDS ears produced significantly larger amplitude oVEMP responses than the normal age-matched control ears and the contralateral ear to the SCDS. In addition, when examining the amplitude of the response within groups (i.e., within SCDS ears, within normal control ears, and within contralateral SCDS ears), the belly-tendon electrode montage resulted in significantly larger amplitude responses than the infraorbital electrode montage for all three groups. The belly-tendon electrode montage produced median increases in the recorded amplitude for all three groups; meaning that for each group, the belly-tendon electrode montage resulted in an increase in amplitude over the infraorbital electrode montage. Specifically, the median increase in oVEMP N1-P1 amplitudes using the belly-tendon montage was $39 \%$ greater in control ears, $76 \%$ greater in the SCDS ears, and $17 \%$ greater in the contralateral SCDS ears.

The average oVEMP response measured in the SCDS ears when using the infraorbital electrode montage was $7.98 \mu \mathrm{V}$. Although this value was significantly larger than the responses measured in the normal ear contralateral to the SCDS ear and the age-matched control ears, it is a much smaller value than what has previously been reported in the literature for oVEMP peak-to-peak amplitude responses when using the infraorbital electrode montage in participants with SCDS. Zuniga et al (2014) reported median peak-to-peak amplitudes of $66.6 \mu \mathrm{V}$ in their SCDS group. A second study by Zuniga et al (2013) reported median peak-to-peak amplitudes of $48.9 \mu \mathrm{V}$ in participants with SCDS. Janky et al (2013) reported

Table 1. N1 and P1 Latency Averages and SDs for Both Electrode Montages and All Three Subgroups

\begin{tabular}{lcc}
\hline & N1 & P1 \\
& Latency (SD) & Latency (SD) \\
\hline Belly-tendon SCDS & $10.49 \mathrm{msec}(0.62)$ & $14.61 \mathrm{msec}(0.62)$ \\
Belly-tendon & $11.23 \mathrm{msec}(0.63)$ & $15.64 \mathrm{msec}(1.57)$ \\
$\quad$ contraSCDS & & \\
Belly-tendon control & $11.13 \mathrm{msec}(0.53)$ & $15.27 \mathrm{msec}(1.24)$ \\
Infraorbital SCDS & $10.76 \mathrm{msec}(0.80)$ & $15.32 \mathrm{msec}(1.01)$ \\
Infraorbital & $11.24 \mathrm{msec}(0.81)$ & $15.92 \mathrm{msec}(1.15)$ \\
$\quad$ contraSCDS & & \\
Infraorbital control & $11.10 \mathrm{msec}(0.59)$ & $15.34 \mathrm{msec}(1.28)$ \\
\hline
\end{tabular}


Table 2. N1-P1 Peak-to-Peak Amplitude Averages and SDs for Both Electrode Montages and All Three Subgroups

\begin{tabular}{lc}
\hline & N1-P1 Amplitude (SD) \\
\hline Belly-tendon SCDS & $28.91 \mu \mathrm{V}(21.47)$ \\
Belly-tendon contraSCDS & $5.75 \mu \mathrm{V}(5.42)$ \\
Belly-tendon control & $5.96 \mu \mathrm{V}(4.68)$ \\
Infraorbital SCDS & $10.08 \mu \mathrm{V}(9.73)$ \\
Infraorbital contraSCDS & $3.24 \mu \mathrm{V}(2.52)$ \\
Infraorbital control & $3.31 \mu \mathrm{V}(1.93)$ \\
\hline
\end{tabular}

mean amplitudes of $29.04 \mu \mathrm{V}$ in their participants with SCDS. The age range and number of participants in all of the aforementioned studies were comparable to the age range and number of participants in the current study, so it is uncertain as to why the infraorbital electrode montage resulted in significantly smaller amplitudes in this dataset than those previously reported.

It should be noted that the variability of the measured amplitude from the belly-tendon electrode montage was much higher than the variability of the response measured from the infraorbital montage within the SCDS groups and when compared with the other groups (i.e., larger SD for the belly-tendon montage within the SCDS group). Sandhu et al (2013) and Vanspauwen et al (2017) also reported increased variability with the belly-tendon electrode montage when measuring oVEMP responses in normal participants. They both attributed this to the active electrode being placed closer to the lateral rectus muscle and the reference electrode being placed closer to the medial rectus muscle. These horizontal rectus muscles (i.e., lateral and medial rectus) were shown by Govender et al (2011) to be activated by the oVEMP stimulus. This activation may impact the purity of the oVEMP reflex and result in greater variability. Despite the variability seen in the amplitude of the response from the belly-tendon electrode montage for this current study, all of the oVEMP responses from the belly-tendon electrode montage were greater in amplitude than those measured from the infraorbital electrode montage. Figure 2 illustrates the variability found in the responses. It also shows the outliers in each condition, however because of the great variability and large SD for the belly-tendon montage in the participants with superior canal dehiscence, all outliers in that condition fall within the SD and are not visible on the figure.

Sandhu et al (2013) found no significant difference in $\mathrm{N} 1$ or P1 latency when using the belly-tendon electrode montage over the other electrode montages; however, there was a trend towards a shorter N1 latency with the belly-tendon electrode montage. Makowiec et al (2017) found a significantly shorter N1 latency when recording oVEMPs with the belly-tendon electrode montage

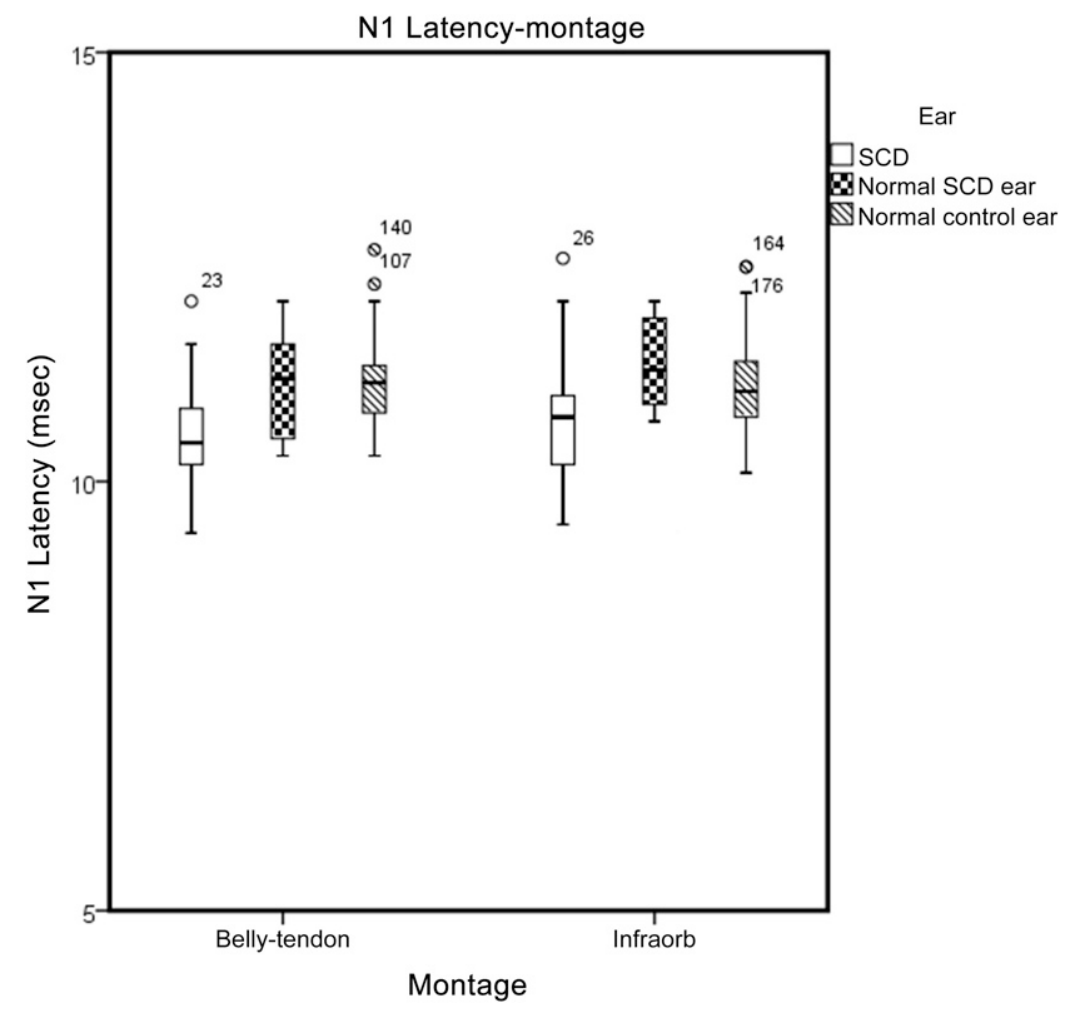

Figure 4. Sample oVEMP waveforms obtained from both electrode montages for all three category groups. Left-hand side $=$ responses from the belly-tendon electrode montage, and right-hand side = responses from the infraorbital electrode montage. Top to bottom: SCDS ear, normal ear contralateral to the SCDS ear, and normal control ear. 


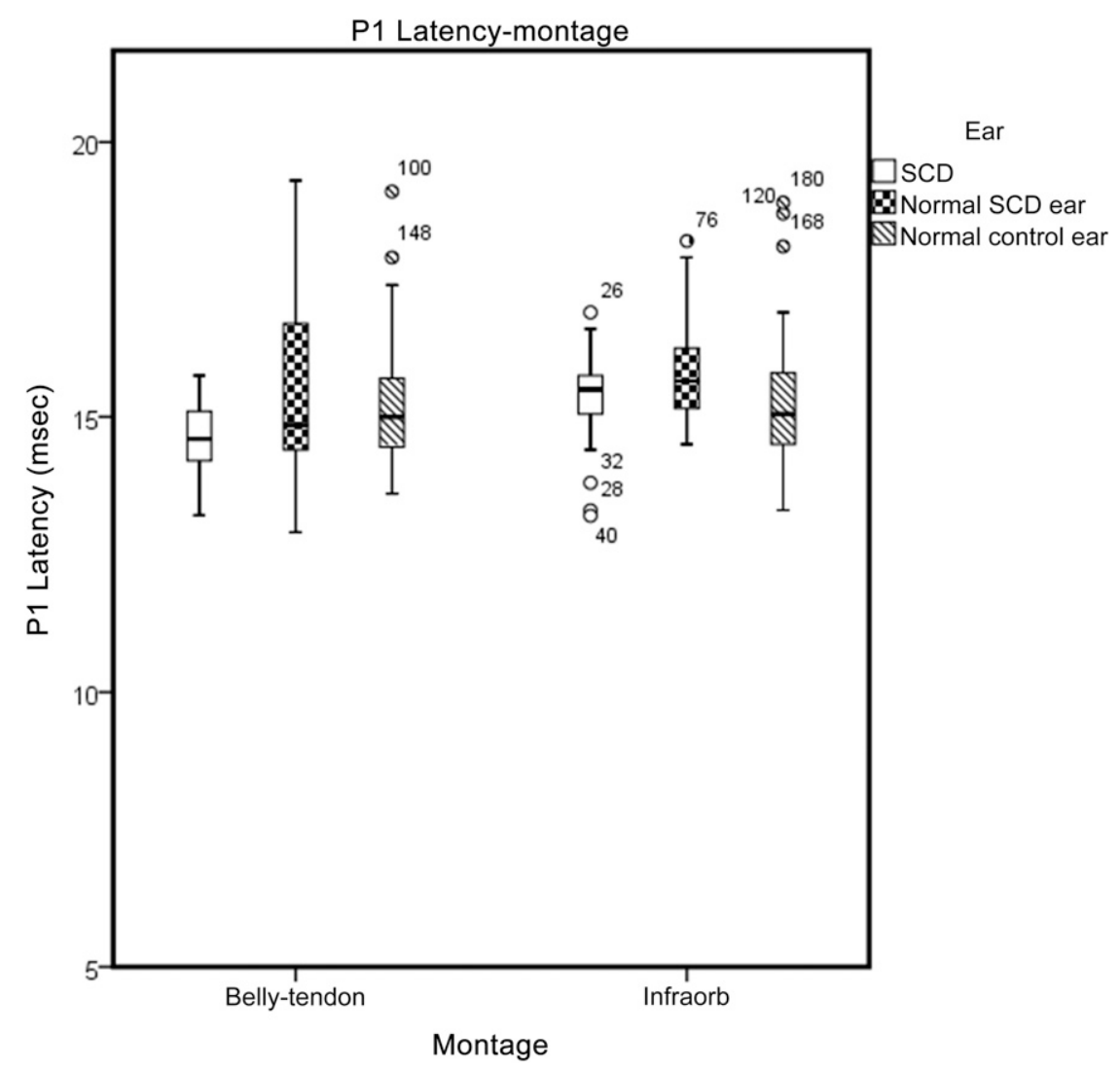

Figure 5. Boxplots of $\mathrm{P} 1$ latency for the two different montages and three groups. The thin line within the box designates the mean. The error bars above and below the box indicate the 90th and 10th percentiles. Outliers are represented by the points outside the boxes.

over the infraorbital electrode montage in young, normal participants. The findings for this study were in agreement with Sandhu et al and found no significant difference in $\mathrm{N} 1$ or P1 latency for either the belly-tendon electrode montage or the infraorbital electrode montage. Both the studies by Sandhu et al (2013) and Makowiec et al (2017) used young participants (i.e., in their 20s and 30s). It is not completely understood as to why Makowiec et al (2017) found a significantly shorter N1 latency than this current study and Sandhu et al (2013). Further research in this area is needed.

The belly-tendon electrode montage increases the amplitude of the oVEMP response by a great percentage, especially in the ear positive for SCDS (i.e., median increase of $76 \%$ in the oVEMP amplitude when using the belly-tendon electrode montage). Increasing the amplitude of the oVEMP response makes it easier to identify clinically as the response is so much larger than the myogenic noise in the recording. Therefore, we suggest that the bellytendon electrode montage is used when oVEMPs are measured clinically.

Future research is needed to examine the impact of the size of the dehiscence on the oVEMP response with the belly-tendon electrode montage. Hunter et al (2016) found a significant interaction of oVEMP amplitude and the size of the dehiscence when the response was measured with the infraorbital electrode montage (i.e., the oVEMP amplitude increased as the size of the dehiscence increased). In this regard, in cases of smaller dehiscence, the bellytendon montage may offer a higher sensitivity. This relationship has yet to be examined for the belly-tendon electrode montage as this study did not control for the size of the dehiscence.

Acknowledgments. The authors are grateful for the assistance in identifying potential study participants provided by Brendan O'Connell, M.D.; Alejandro Rivas, M.D.; Timothy Trone, M.D.; and Kenneth Watford, D.NP.

\section{REFERENCES}

Belden C, Weg N, Minor L, Zinreich S. (2003) CT evaluation of bone dehiscence of the superior semicircular canal as a cause of sound- and/or pressure-induced vertigo. Radiology 226(2):337-343.

Brantberg K, Bergenius J, Tribukait A. (1999) Vestibular-evoked myogenic potentials in patients with dehiscence of the superior semicircular canal. Acta Otolaryngol 119(6):633-640.

Chihara Y, Iwasaki S, Ushio M, Murofushi T. (2007) Vestibularevoked extraocular potentials by air-conducted sound: another clinical test for vestibular function. Clin Neurophysiol 118:2745-2751.

Colebatch J, Halmagyi G, Skuse N. (1994) Myogenic potentials generated by a click-evoked vestibulocollic reflex. J Neurol Neurosurg Psychiatry 57(2):190-197. 
Curthoys I, Iwasaki S, Chihara Y, Ushio M, McGarvie L, Burgess A. (2011) The ocular vestibular evoked myogenic potential to air conducted sound; probable superior vestibular nerve origin. Clin Neurophysiol 122(3):611-616.

Dumitru D, DeLisa L. (1991) AAEM minimonograph 10: volume conduction. Muscle Nerve 14:605-624.

Govender S, Rosengren S, Colebatch J. (2009) The effect of gaze direction on the ocular vestibular evoked myogenic potential produced by air-conducted sound. Clin Neurophysiol 120:1386-1391.

Govender S, Rosengren S, Colebatch J. (2011) Ocular vestibular evoked myogenic potentials produced by impulsive lateral acceleration in unilateral vestibular dysfunction. Clin Neurophysiol 122:2498-2504.

Hunter J, O'Connell B, Wang J, Chakravorti S, Makowiec K, Carlson M, Dawant B, McCaslin D, Noble J, Wanna G. (2016) Correlation of superior canal dehiscence surface area with vestibular evoked myogenic potentials, audiometric thresholds, and dizziness handicap. Otol Neorutol 37(8):1104-1110.

Iwasaki S, Chihara Y, Smulders Y, Burgess A, Halmagyi G, Curthoys I, Murofushi T. (2009) The role of the superior vestibular nerve in generating ovular vestibular evoked myogenic potentials to bone conducted vibration at Fz. Clin Neurophysiol 120:588-593.

Iwasaki S, McGarvie L, Halmagyi G, Burgess A, Kim J, Colebatch J, Curthoys I. (2007) Head taps evoke a crossed vestibulo-ocular reflex. Neurology 68:1227-1229.

Janky K, Nguyen K, Welgampola M, Zuniga M, Carey J. (2013) Air-conducted oVEMPs provide the best separation between intact and superior canal dehiscent labyrinths. Otol Neurotol 34(1):127-134.

Makowiec K, McCaslin D, Jacobson G, Hatton K, Lee J. (2017) Effect of electrode montage and head position on air conducted ocular vestibular evoked myogenic potential (oVEMP). Am J Audiol 26(2):180-188.

Manzari L, Tedesco A, Burgess A, Curthoys I. (2010) Ocular vestibular evoked myogenic potentials to bone conducted vibration in superior vestibular neuritis show utricular function. Otolaryngol Head Neck Surg 143:274-280.

Minor L, Solomon D, Zinreich J, Zee D. (1998) Sound- and/or pressureinduced vertigo due to bone dehiscence of the superior semicircular canal. Arch Otololaryngol Head Neck Surg 124(3):249-258.

Murnane O, Akin F, Kelly J, Byrd S. (2011) Effects of stimulus and recording parameters on the air conduction ocular vestibular evoked myogenic potential. J Am Acad Audiol 22:469-480.

Piker E, Jacobson G, McCaslin D, Hood L. (2011) Normal characteristics of the ocular vestibular evoked myogenic potential. J Am Acad Audiol 22(4):222-230.
Rosengren S, Jombik P, Halmagyi G, Colebatch J. (2009) Galvanic ocular vestibular evoked myogenic potentials provide new insight into vestibulo-ocular reflexes and unilateral vestibular loss. Clin Neurophysiol 120:569-580.

Rosengren S, McAngus T, Colebach J. (2005) Vestibular evoked extraocular potentials produced by stimulation with bone-conducted sound. Clin Neurophysiol 116(8):1938-1948.

Rosengren S, Welgampola M, Colebatch J. (2010) Vestibular evoked myogenic potentials: past, present and future. Clin Neurophysiol 121:636-651.

Sandhu J, George S, Rea P. (2013) The effect of electrode positioning on the ocular vestibular evoked myogenic potential to air-conducted sound. Clin Neurophysiol 124(6):1232-1236.

Shojaku H, Watanabe Y, Tsubota M, Katayama N. (2008) Evaluation of the vestibular evoked myogenic potential during parabolic flight in humans. Exp Brain Res 187:477-481.

Suzuki J, Tokumasi K, Goto K. (1969) Eye movements from single utricular nerve stimulation in the cat. Acta Otolaryngol 68: $350-362$.

Taylor R, Xing M, Black D, Halmagyi G, Welgampola M. (2015) Ocular vestibular evoked myogenic potentials: the effect of head and body tilt in the roll plane. Clin Neurophysiol 125: 627-634.

Todd N, Rosengren S, Aw S, Colebatch J. (2007) Ocular vestibular evoked myogenic potentials (OVEMPs) produced by air- and boneconducted sound. Clin Neurophysiol 118:381-390.

Vanspauwen R, Wuyts F, Krijger S, Maes L. (2017) Comparison of different electrode configurations for the oVEMP with bone-conducted vibration. Ear Hear 38(2):205-211.

Wang S, Tseng C, Young Y. (2014) Selective effects of head posture on ocular vestibular-evoked myogenic potential (oVEMP) by boneconduction vibration. Clin Neurophysiol 125:621-626.

Welgampola M, Carey J. (2010) Waiting for the evidence: VEMP testing and the ability to differentiate utricular versus saccular function. Otolaryngol Head Neck Surg 143(2):281-283.

Zuniga M, Davalos-Bichara M, Schubert M, Carey J, Janky K. (2014) Optimizing ocular vestibular evoked myogenic potential testing for superior semicircular canal dehiscence syndrome: electrode placement. Audiol Neurol 19:239-247.

Zuniga M, Janky K, Nguyen K, Welgampola M, Carey J. (2013) Ocular vs. cervical VEMPs in the diagnosis of superior semicircular canal dehiscence syndrome. Otol Neurotol 34(1): $121-126$ 